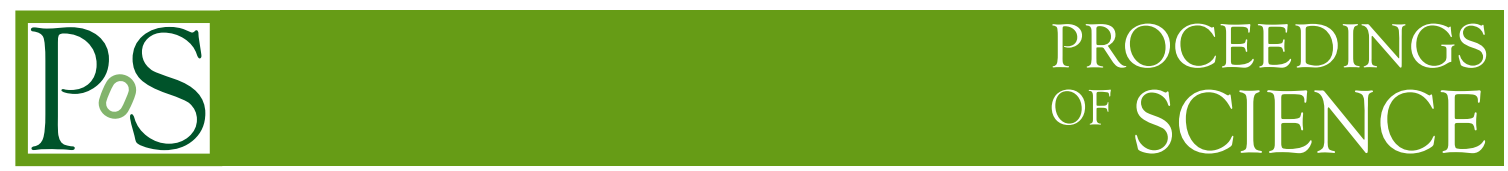

\title{
CUORE: first results and prospects
}

\author{
V. Novati ${ }^{* 24}$, C. Alduino, ${ }^{1}$ K. Alfonso ${ }^{2}$, F. T. Avignone III $^{1}$, O. Azzolini ${ }^{3}$, G. Bari ${ }^{4}$, \\ F. Bellini ${ }^{5,6}$, G. Benato ${ }^{7}$, A. Bersani ${ }^{8}$, M. Biassoni ${ }^{9}$, A. Branca ${ }^{10,11}$, C. Brofferio ${ }^{12,9}$, \\ C. Bucci ${ }^{13}$, A. Camacho ${ }^{3}$, A. Caminata ${ }^{8}$, L. Canonica ${ }^{14,13}$, X. G. Cao ${ }^{15}$, S. Capelli ${ }^{12,9}$, \\ L. Cappelli ${ }^{7,16,13}$, L. Cardani ${ }^{6}$, P. Carniti ${ }^{12,9}$, N. Casali ${ }^{6}$, L. Cassina ${ }^{12,9}$, D. Chiesa ${ }^{12,9}$, \\ N. Chott ${ }^{1}$, M. Clemenza ${ }^{12,9}$, S. Copello ${ }^{17,8}$, C. Cosmelli ${ }^{5,6}$, O. Cremonesi ${ }^{9}$, \\ R. J. Creswick ${ }^{1}$, J. S. Cushman ${ }^{18}$, A. D'Addabbo ${ }^{13}$, D. D'Aguanno ${ }^{13,19}$, I. Dafinei ${ }^{6}$, \\ C. J. Davis ${ }^{18}$, S. Dell'Oro ${ }^{20}$, M. M. Deninno ${ }^{4}$, S. Di Domizio ${ }^{17,8}$, M. L. Di Vacri ${ }^{13,21}$, \\ V. Dompè ${ }^{13,22}$, A. Drobizhev ${ }^{7,16}$, D. Q. Fang ${ }^{15}$, M. Faverzani ${ }^{12,9}$, E. Ferri' ${ }^{9}$, F. Ferroni ${ }^{5,6}$, \\ E. Fiorini ${ }^{9,12}$, M. A. Franceschi ${ }^{23}$, S. J. Freedman ${ }^{16,7 \dagger}$, B. K. Fujikawa ${ }^{16}$, \\ A. Giachero ${ }^{12,9}$, L. Gironi ${ }^{12,9}$, A. Giuliani ${ }^{24}$, L. Gladstone ${ }^{14}$, P. Gorla ${ }^{13}$, C. Gotti ${ }^{12,9}$, \\ T. D. Gutierrez ${ }^{25}$, K. Han $^{26}$, K. M. Heeger ${ }^{18}$, R. Hennings-Yeomans ${ }^{7,16}$, H. Z. Huang ${ }^{2}$, \\ G. Keppel ${ }^{3}$, Yu. G. Kolomensky ${ }^{7,16}$, A. Leder ${ }^{14}$, C. Ligi' ${ }^{23}$, K. E. Lim ${ }^{18}$, Y. G. Ma15, \\ L. Marini ${ }^{17,8}$, M. Martinez ${ }^{5,6,27}$, R. H. Maruyama ${ }^{18}$, Y. Mei ${ }^{16}$, N. Moggi ${ }^{28,4}$, S. Morganti ${ }^{6}$, \\ S. S. Nagorny ${ }^{13,22}$, T. Napolitano ${ }^{23}$, M. Nastasi ${ }^{12,9}$, C. Nones ${ }^{29}$, E. B. Norman ${ }^{30,31}$, \\ A. Nucciotti ${ }^{12,9}$, I. Nutini ${ }^{13,22}$, T. O'Donnell ${ }^{20}$, J. L. Ouellet ${ }^{14}$, C. E. Pagliarone ${ }^{13,19}$, \\ M. Pallavicini ${ }^{17,8}$, V. Palmieri ${ }^{3}$, L. Pattavina ${ }^{13}$, M. Pavan ${ }^{12,9}$, G. Pessina ${ }^{9}$, C. Pira ${ }^{3}$, \\ S. Pirro ${ }^{13}$, S. Pozzi ${ }^{12,9}$, E. Previtali ${ }^{9}$, F. Reindl ${ }^{6}$, C. Rosenfeld ${ }^{1}$, C. Rusconi ${ }^{1,13}$, \\ M. Sakai ${ }^{2}$, S. Sangiorgio ${ }^{30}$, D. Santone ${ }^{13,21}$, B. Schmidt ${ }^{16}$, J. Schmidt ${ }^{2}$,

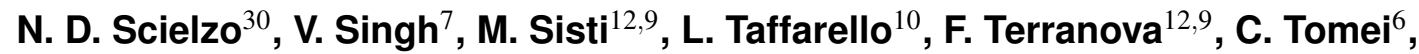 \\ M. Vignati ${ }^{6}$, S. L. Wagaarachchi ${ }^{7,16}$, B. S. Wang ${ }^{30,31}$, H. W. Wang ${ }^{15}$, B. Welliver ${ }^{16}$, \\ J. Wilson ${ }^{1}$, K. Wilson ${ }^{1}$, L. A. Winslow ${ }^{14}$, T. Wise ${ }^{18,32}$, L. Zanotti ${ }^{12,9}$, G. Q. Zhang ${ }^{15}$, \\ S. Zimmermann ${ }^{33}$, S. Zucchelli ${ }^{28,4}$
}

\footnotetext{
${ }^{1}$ Department of Physics and Astronomy, University of South Carolina, Columbia, SC 29208, USA

${ }^{2}$ Department of Physics and Astronomy, University of California, Los Angeles, CA 90095, USA

${ }^{3}$ INFN - Laboratori Nazionali di Legnaro, Legnaro (Padova) I-35020, Italy

${ }^{4}$ INFN - Sezione di Bologna, Bologna I-40127, Italy

${ }^{5}$ Dipartimento di Fisica, Sapienza Università di Roma, Roma I-00185, Italy

${ }^{6}$ INFN - Sezione di Roma, Roma I-00185, Italy

${ }^{7}$ Department of Physics, University of California, Berkeley, CA 94720, USA

${ }^{8}$ INFN - Sezione di Genova, Genova I-16146, Italy

${ }^{9}$ INFN - Sezione di Milano Bicocca, Milano I-20126, Italy

${ }^{10}$ INFN - Sezione di Padova, Padova I-35131, Italy

${ }^{11}$ Dipartimento di Fisica e Astronomia, Università di Padova, I-35131 Padova, Italy
} 
${ }^{12}$ Dipartimento di Fisica, Università di Milano-Bicocca, Milano I-20126, Italy

${ }^{13}$ INFN - Laboratori Nazionali del Gran Sasso, Assergi (L'Aquila) I-67100, Italy

${ }^{14}$ Massachusetts Institute of Technology, Cambridge, MA 02139, USA

${ }^{15}$ Shanghai Institute of Applied Physics, Chinese Academy of Sciences, Shanghai 201800, China

${ }^{16}$ Nuclear Science Division, Lawrence Berkeley National Laboratory, Berkeley, CA 94720, USA

${ }^{17}$ Dipartimento di Fisica, Università di Genova, Genova I-16146, Italy

${ }^{18}$ Wright Laboratory, Department of Physics, Yale University, New Haven, CT 06520, USA

${ }^{19}$ Dipartimento di Ingegneria Civile e Meccanica, Università degli Studi di Cassino e del Lazio Meridionale, Cassino I-03043, Italy

${ }^{20}$ Center for Neutrino Physics, Virginia Polytechnic Institute and State University, Blacksburg, Virginia 24061, USA

${ }^{21}$ Dipartimento di Scienze Fisiche e Chimiche, Università dell'Aquila, L'Aquila I-67100, Italy

${ }^{22}$ INFN - Gran Sasso Science Institute, L'Aquila I-67100, Italy

${ }^{23}$ INFN - Laboratori Nazionali di Frascati, Frascati (Roma) I-00044, Italy

${ }^{24}$ CSNSM, Univ. Paris-Sud, CNRS/IN2P3, Université Paris-Saclay, Orsay 91405, France

${ }^{25}$ Physics Department, California Polytechnic State University, San Luis Obispo, CA 93407, USA

${ }^{26}$ INPAC and School of Physics and Astronomy, Shanghai Jiao Tong University; Shanghai Laboratory for Particle Physics and Cosmology, Shanghai 200240, China

${ }^{27}$ Laboratorio de Fisica Nuclear y Astroparticulas, Universidad de Zaragoza, Zaragoza 50009, Spain

${ }^{28}$ Dipartimento di Fisica e Astronomia, Alma Mater Studiorum - Università di Bologna, Bologna I-40127, Italy

${ }^{29}$ IRFU, CEA, Université Paris-Saclay, 91191 Gif-sur-Yvette, France

${ }^{30}$ Lawrence Livermore National Laboratory, Livermore, CA 94550, USA

${ }^{31}$ Department of Nuclear Engineering, University of California, Berkeley, CA 94720, USA

${ }^{32}$ Department of Physics, University of Wisconsin, Madison, WI 53706, USA

${ }^{33}$ Engineering Division, Lawrence Berkeley National Laboratory, Berkeley, CA 94720, USA

E-mail: valentina.novatiecsnsm.in2p3.fr

CUORE is the first bolometric tonne-scale experiment aiming at the investigation of neutrinoless double-beta $(0 v 2 \beta)$ decay of ${ }^{130} \mathrm{Te}$. The cryogenic commissioning followed by the detector installation and cool down took place during 2016. After the optimisation of all the detectors, the data-taking started in spring 2017. We report about the results of the first dataset acquired in May, which led to a limit on the $0 v 2 \beta$ half-life of ${ }^{130} \mathrm{Te}$ of $6.6 \times 10^{24} \mathrm{yr}$. An upgrade of CUORE, named CUPID, is planned to improve the $0 v 2 \beta$-decay sensitivity via passive and active background reduction and crystal enrichment. Some technologies for CUPID are currently under study and two of them are presented here, involving the detection of Cherenkov and scintillation light emitted by enriched ${ }^{130} \mathrm{TeO}_{2}$ and $\mathrm{Li}_{2}^{100} \mathrm{MoO}_{4}$ crystals respectively. This will allow us to reject the currently dominant $\alpha$ background.

The 19th International Workshop on Neutrinos from Accelerators-NUFACT2017

25-30 September, 2017

Uppsala University, Uppsala, Sweden

\footnotetext{
*Speaker.
}

${ }^{\dagger}$ Deceased. 


\section{Introduction}

Neutrinoless double-beta $(0 v 2 \beta)$ decay [1] is a rare hypothetical nuclear transition, which consists in the transformation of two neutrons in two protons and two electrons with no neutrinos in the final state. This process is possible only if the neutrino is a Majorana particle, more in general its observation would demonstrate the non-conservation of the lepton number [1]. If mediated by the exchange of light Majorana neutrinos, the discovery of this process would fix the neutrino mass-scale, would contribute to the definition of the neutrino mass hierarchy, and in general to a deeper knowledge of the neutrino itself [1].

CUORE (Cryogenic Underground Observatory for Rare Events) experiment [2] is the first tonne-scale experiment based on bolometers searching the $0 v 2 \beta$ decay of the ${ }^{130} \mathrm{Te}$, whose expected signal is a peak at the $Q$-value of the transition $(2527 \mathrm{keV})$. This isotope is a good candidate for $0 v 2 \beta$ decay thanks to its high isotopic abundance (34.2\%). It is embedded in the detectors themselves, which consist of 5-cm-side cubic $\mathrm{TeO}_{2}$ crystals with a mass of $750 \mathrm{~g}$. This well-known compound has been chosen because of its excellent bolometric performance ( $\sim 5 \mathrm{keV}$ FWHM energy resolution at the $2615 \mathrm{keV}{ }^{208} \mathrm{Tl} \gamma$ quanta [3]) and crystals radiopurity $\left({ }^{232} \mathrm{Th}<0.8 \mu \mathrm{Bq} / \mathrm{kg}\right.$, ${ }^{238} \mathrm{U}<0.7 \mu \mathrm{Bq} / \mathrm{kg}$ assuming secular equilibrium $\left.[4,5]\right)$. CUORE is using 988 bolometers arranged in 19 towers constituted by 13 floors of 4 detectors each. Each crystal is equipped with a neutron-transmutation-doped germanium (NTD-Ge) thermistor for the read-out of the temperature signal and a silicon heater used for the thermal gain stabilisation. The experiment is hosted at LNGS (Laboratori Nazionali del Gran Sasso, Italy) in a liquid-helium-free cryostat, which can reach $10 \mathrm{mK}$ temperature with the combined use of a diluition unit and four pulse tubes.

\section{CUORE first results}

After the cryogenic system commissioning, the CUORE detector was installed during summer 2016 and then cooled down to base temperature at the end of the same year. This work reports about the results obtained from the first dataset, corresponding to a 3-week measurement, acquired in May 2017 after a preliminary optimisation of the detector. 984 detectors are working and $90 \%$ of them have been used in this analysis, providing a total ${ }^{130} \mathrm{Te}$ exposure of $10.6 \mathrm{~kg} \cdot \mathrm{yr}$. We considered a region of interest (ROI) between 2465 and $2575 \mathrm{keV}$ and a total efficiency of $55.3 \pm 3 \%$ (including trigger and energy reconstruction, pulse shape discrimination, single-hit events and $0 v 2 \beta$ containment). The recorded background, $9.8_{-1.5}^{+1.7} \times 10^{-3}$ counts/(keV kg yr), showed a good agreement with the Monte Carlo simulations [6]. Fig. 1 shows the improvement in the backgound level from CUORE-0 to CUORE: the few events present in the ROI are mainly due to degraded-energy $\alpha$ particles emitted by detector surfaces [5]. Energy spectra show a good energy resolution: $10.6 \mathrm{keV}$ FWHM at $2615 \mathrm{keV}$ of ${ }^{208} \mathrm{Tl} \gamma$ quanta were obtained during the first calibration and they were then improved to $7.9 \mathrm{keV}$ during the physics run thanks to the lower pile-up rate. Ongoing studies and optimisation are aiming to a further improvement of the energy resolution of the detector. Fig. 1 shows the background spectrum in the vicinity of the ROI and the fit used to obtain the $0 v 2 \beta$ half-life limit: $T_{1 / 2}^{0 v 2 \beta}>4.5 \times 10^{24} \mathrm{yr}$ at $90 \%$ C.L. This result, combined with the limits obtained by CUORICINO (19.75 kg.yr) and CUORE-0 (9.8 kg.yr), produces a more stringent limit of $T_{1 / 2}^{0 v 2 \beta}$ 

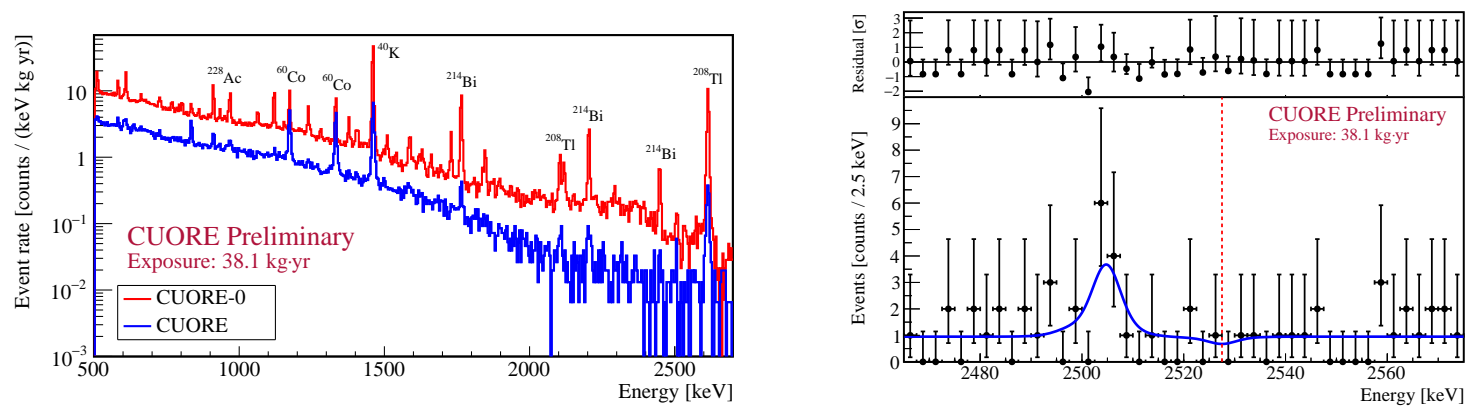

Figure 1: Left: Comparison of background spectra in the $\gamma$ region for CUORE-0 and CUORE experiments. Right: Best fit in the ROI with residuals. The peak at $(2504.8 \pm 1.2) \mathrm{keV}$ is a sum $\gamma$ line from ${ }^{60} \mathrm{Co}$.

$>6.6 \times 10^{24} \mathrm{yr}$ at $90 \%$ C.L. ${ }^{1}$. CUORE will run for 5 years and its final sensitivity on $T_{1 / 2}^{0 v 2 \beta}$ will be $9 \times 10^{25} \mathrm{yr}$ [8]. This new limit will start to probe the inverted hierarchy region of neutrino masses.

\section{CUPID experiment}

An upgrade of CUORE is foreseen at the end of its data taking. This new experiment is named CUPID (CUORE Upgrade with Particle IDentification) [9], and its purpose will be to investigate the entire inverted hierarchy region, reaching a sensitivity to the $0 v 2 \beta$ half-life of the order of $10^{27}-10^{28} \mathrm{yr}$. This goal will be achieved by increasing the number of $2 \beta$-active nuclei (thanks to crystal enrichment) and by reducing the background. The CUORE main $\alpha$ background can be removed with the help of particle identification. One of the most promising active background rejection technique considered for CUPID [10] is the use of bolometric light detector to register the Cherenkov or scintillation radiation emitted by the main absorber. Some technologies are presently under test $[11,12,13,14,15,16,17,18,19,20]$ to find the best solution in view of CUPID. Two of them, based on ${ }^{130} \mathrm{TeO}_{2}$ and $\mathrm{Li}_{2}^{100} \mathrm{MoO}_{4}$ crystals, are illustrated below.

$\mathrm{TeO}_{2}$ crystals emit a feeble light that can be used to tag $\beta(\gamma)$ events. The light has two origins: it is mainly produced by Cherenkov effect [21], but a non-negligible component of scintillation is also present [15]. The Cherenkov threshold is $50 \mathrm{keV}$ for $\beta(\gamma)$ and $400 \mathrm{MeV}$ for $\alpha$, which can be rejected when their thermal energy populates the ROI. A $\beta$-like event in the $0 v 2 \beta$ region corresponds to an energy of only $100 \mathrm{eV}$ in terms of collected light and the related signal is difficult to observe with a standard light detector based on NTD-technology [11, 17]. This challenge can be faced by a light detector boosted by the Neganov-Luke effect [22, 23]: this kind of devices manage to amplify thermal signals thanks to an electric field, consequently improving the signal-to-noise ratio and lowering the threshold $[12,13,14,15]$. Two Neganov-Luke-assisted light detectors, coupled to two enriched ${ }^{130} \mathrm{TeO}_{2}$ crystals (435 g) at LNGS, allowed us to separate $\beta(\gamma)$ from $\alpha \mathrm{s}$ obtaining an $\alpha$ rejection factor of $98.21 \%$ and $99.99 \%$ with a $\beta(\gamma)$ acceptance of $95 \%$ [14]. Also, the two bolometers showed a high energy resolution ( 6.5 and $4.3 \mathrm{keV} \mathrm{FWHM}$ at the $2615 \mathrm{keV} \gamma$ quanta of $\left.{ }^{208} \mathrm{Tl}\right)$ and a high radiopurity $\left({ }^{228} \mathrm{Th}\right.$ and $\left.{ }^{226} \mathrm{Ra}<3.1 \mu \mathrm{Bq} / \mathrm{kg}\right)$.

An interesting alternative for the CUPID experiment is to change the $0 v 2 \beta$-decay isotope: ${ }^{100} \mathrm{Mo}$ is a promising candidate thanks to its $Q$-value $(3034 \mathrm{keV})$ higher than the natural $\gamma$ ra-

\footnotetext{
${ }^{1}$ This dataset has been reprocessed and combined with a new physics run acquired in August 2017 [7].
} 
dioactivity and its reasonably high isotopic abundance of $9.7 \%$ combined with the possibility of enrichment above $95 \%$. A favorable compound containing ${ }^{100} \mathrm{Mo}$ is $\mathrm{Li}_{2} \mathrm{MoO}_{4}$, developed by the LUMINEU project $[19,20]$. Detectors based on this crystal scintillator have a high energy resolution in the ROI $(<6 \mathrm{keV})$, are characterised by an excellent radiopurity $\left(<6 \mu \mathrm{Bq} / \mathrm{kg}\right.$ for ${ }^{228} \mathrm{Th}$ and $<11 \mu \mathrm{Bq} / \mathrm{kg}$ for ${ }^{226} \mathrm{Ra}$ ) and are able to fully separate $\alpha \mathrm{s}(>9 \sigma)$ thanks to an adequate scintillation efficiency. Four enriched $\mathrm{Li}_{2}^{100} \mathrm{MoO}_{4}$ crystals have been tested underground at the LSM (Laboratoire Souterrain de Modane, France), showing a good reproducibility [20]. The next step will be the measurement of an array consisting of $20 \mathrm{Li}_{2}^{100} \mathrm{MoO}_{4}$ bolometers that will start taking data in early 2018 at LSM. In view of CUPID, a capacity for crystal mass production, possibly multi-site, will be crucial: the present $\mathrm{Li}_{2}^{100} \mathrm{MoO}_{4}$ crystal growth has been performed at Nikolaev Institute of Inorganic Chemistry (NIIC, Novosibirsk, Russia) but new possibilities are under study in France (CLYMENE project [24]) and in China (CUPID-China).

\section{Conclusions and acknowledgements}

CUORE, the first tonne-scale cryogenic experiment based on $\mathrm{TeO}_{2}$ bolometers, successfully accomplished the commissioning in 2016, the first dataset was acquired during May 2017 and was followed by the first data release in July 2017. A preliminary analysis of this first dataset, combined with the previous limits of CUORICINO and CUORE-0 experiments, set a limit on the $0 v 2 \beta$ half-life to $6.6 \times 10^{24} \mathrm{yr}$ with a sensitivity of $9 \times 10^{25} \mathrm{yr}$ in 5 years of data-taking. The present performance and results of two technologies developed in view of the CUPID experiment have been reported. The feasibility to measure the tiny light emitted by enriched $\mathrm{TeO}_{2}$ crystals, guaranteeing the rejection of the $\alpha$ background, has been demonstrated. Scintillating $\mathrm{Li}_{2}^{100} \mathrm{MoO}_{4}$ bolometers confirmed their performance reproducibility in terms of radiopurity, energy resolution and $\alpha / \beta(\gamma)$ separation. A 20-crystal pilot experiment (CUPID-Mo) with a significant isotope mass of about $2.5 \mathrm{~kg}$ will start taking data in 2018 .

The CUORE Collaboration thanks the directors and staff of the Laboratori Nazionali del Gran Sasso and the technical staff of our laboratories. This work was supported by the Istituto Nazionale di Fisica Nucleare, the National Science Foundation, the Alfred P. Sloan Foundation, the University of Wisconsin Foundation, and Yale University. This material is also based upon work supported by the US Department of Energy Office of Science, Office of Nuclear Physics. This research used resources of the National Energy Research Scientific Computing Center. The work on luminescent bolometers was supported by LUMINEU, receiving founds from the Agence Nationale de la Recherche (France).

\section{References}

[1] J. D. Vergados et al., Neutrinoless double beta decay and neutrino mass, Int. J. Mod. Phys. E 25 (2016) 1630007.

[2] CUORE collaboration, D. R. Artusa et al., Searching for Neutrinoless Double-Beta Decay of ${ }^{130}$ Te with CUORE, AHEP 2015 (2015) 13.

[3] CUORE collaboration, C. Alduino et al., CUORE-O detector: design, construction and operation, J. Instrum. 11 (2016) P07009. 
[4] CUORE collaboration, F. Alessandria et al., CUORE crystal validation runs: Results on radioactive contamination and extrapolation to CUORE background, Astropart. Phys. 35 (2012) 839.

[5] CUORE collaboration, C. Alduino et al., Measurement of the two-neutrino double-beta decay half-life of Te with the CUORE-O experiment, Eur. Phys. J. C 77 (2017) 13.

[6] CUORE collaboration, C. Alduino et al., The projected background for the CUORE experiment, Eur. Phys. J. C 77 (2017) 543.

[7] CUORE collaboration, C. Alduino et al., First Results from CUORE: A Search for Lepton Number Violation via $0 v \beta \beta$ Decay of ${ }^{130}$ Te, arXiv preprint: 1710.07988 (2017).

[8] CUORE collaboration, C. Alduino et al., CUORE sensitivity to Ov $\beta \beta$ decay, Eur. Phys. J. C 77 (2017) 532.

[9] CUPID collaboration, G. Wang et al., CUPID: CUORE (Cryogenic Underground Observatory for Rare Events) Upgrade with Particle IDentification, arXiv preprint: 1504.03599 (2015) .

[10] CUPID collaboration, G. Wang et al., R\&D towards CUPID (CUORE Upgrade with Particle IDentification), arXiv preprint: 1504.03612 (2015) .

[11] N. Casali et al., $\mathrm{TeO}_{2}$ bolometers with Cherenkov signal tagging: towards next-generation neutrinoless double-beta decay experiments, Eur. Phys. J. C 75 (2015) 12.

[12] L. Pattavina et al., Background Suppression in Massive TeO $\mathrm{O}_{2}$ Bolometers with Neganov-Luke Amplified Light Detectors, J. Low Temp. Phys. 184 (2016) 286.

[13] L. Gironi et al., Cerenkov light identification with Si low-temperature detectors with sensitivity enhanced by the Neganov-Luke effect, Phys. Rev. C 94 (2016) 054608.

[14] D. R. Artusa et al., Enriched $\mathrm{Te}_{2}$ bolometers with active particle discrimination: Towards the CUPID experiment, Phys. Lett. B 767 (2017) 321.

[15] L. Bergé et al., Complete event-by-event $\alpha / \gamma(\beta)$ separation in a full-size TeO $\mathrm{O}_{2} \mathrm{CUORE}$ bolometer by simultaneous heat and light detection, arXiv preprint: 1710.03459 (2017) .

[16] E. S. Battistelli et al., CALDER: neutrinoless double-beta decay identification in $\mathrm{TeO}_{2}$ bolometers with kinetic inductance detectors, Eur. Phys. J. C 75 (2015) 353.

[17] D. Artusa et al., First array of enriched $\mathrm{Zn}^{82}$ Se bolometers to search for double beta decay, Eur. Phys. J. C 76 (2016) 364.

[18] A. S. Barabash et al., First test of an enriched ${ }^{116} \mathrm{CdWO}$ scintillating bolometer for neutrinoless double-beta-decay searches, Eur. Phys. J. C 76 (2016) 487.

[19] E. Armengaud et al., Development of ${ }^{100}$ Mo-containing scintillating bolometers for a high-sensitivity neutrinoless double-beta decay search, Eur. Phys. J. C 77 (2017) 785.

[20] LUMINEU, EDELWEISS, CUPID-0/Mo collaboration, D. V. Poda, ${ }^{100} \mathrm{Mo}_{\text {-enriched } \mathrm{Li}_{2} \mathrm{MoO}_{4}}$ scintillating bolometers for $0 \mathrm{v} 2 \beta$ decay search: From LUMINEU to CUPID-O/Mo projects, AIP Conf. Proc. 1894 (2017) 020017.

[21] T. Tabarelli de Fatis, Cerenkov emission as a positive tag of double beta decays in bolometric experiments, Eur. Phys. J. C 65 (2010) 359.

[22] B. Neganov and V. Trofimov, USSR patent no 1037771, Otkrytia i izobreteniya 146 (1985) 215.

[23] P. N. Luke, Voltage-assisted calorimetric ionization detector, J. Appl. Phys. 64 (1988) 6858.

[24] M. Velázquez et al., Exploratory growth in the $\mathrm{Li}_{2} \mathrm{MoO}_{4}-\mathrm{MoO}_{3}$ system for the next crystal generation of heat-scintillation cryogenic bolometers, Solid State Sci. 65 (2017) 41. 